\title{
Suitability Evaluation of Urban Construction Land in Xi'an Based on Ecological Civilization
}

\author{
Defang Zhao \\ School of Biological and Environmental Engineering, Xi'an University, Shaanxi, China, 710065
}

Keywords: Construction land; ecological civilization; suitability, indicator system model

Abstract: Taking urban construction land in Xi'an as an example, this paper mainly studies the evaluation index system and evaluation model of the suitability evaluation by the GIS technology. And then it studies the quantitative analysis method of land ecological suitability. By building an ecological suitability evaluation index system, this paper mainly USES the CA-MARKOV model to simulate the dynamic of construction land. Finally, the multi-factor layer was extracted, such as of forest land, cultivated land, water body and construction land in Xi'an. The results of Boolean superposition analysis were obtained by selecting the linear combination method of weights. Scope of application: The suitability of Xi'an is $44.34 \%$, the suitable land is $33.86 \%$, the medium suitability is $19.27 \%$, and the suitability is worse than $2.49 \%$. According to the results of ecological suitability evaluation, the characteristics of construction land in Xi'an were evaluated objectively, and suggestions for ecological civilization construction and urban planning and construction were put forward.

\section{Introduction}

Land resources are the foundation of human survival and development, and the material basis for survival and development. Rational use of land resources is an important part of ecological civilization construction. With the acceleration of urban integration, the demand for land is increasing. So, there are many factors that can provide a reliable basis for optimizing the layout of cities and towns by analyzing the suitability of construction land.

\section{Research area overview}

Xi'an City is located in the central part of Shaanxi Province, bordering on the Weihe River and the Loess Plateau in the north and Qinling in the south. Between $107^{\circ} 40^{\prime} \sim 109^{\circ} 49^{\prime}$ east longitude and $33^{\circ} 42^{\prime} \sim 34^{\circ} 45^{\prime}$ north latitude, the altitude difference in the territory is very different. The highest altitude is 2800 meters and the lowest is 345 meters. The east-west length is about 204 kilometers, and the north-south width is about 116 kilometers. Xi'an belongs to the temperate continental semi-humid monsoon climate. The annual precipitation is between 312.2 and $903.2 \mathrm{~mm}$, and the annual average temperature is $13.4^{\circ} \mathrm{C}$. Xi'an is rich in mineral resources. There are 47 mineral resources discovered, mainly distributed in the southern Qinling area, and Xi'an has Good geological conditions for storing geothermal water, only the area of geothermal energy that can be 
developed in urban areas is as high as $780 \mathrm{~km}^{2}$, and the reserves are about 539 million m3. The soil type is rich, and the difference between the north and the south is obvious. The Weihe plain in the north is mainly fluvo-aquic soil, paddy soil, etc. The land is fertile and the land resources are relatively abundant. The southern Qinling mountains are mostly native soils, mainly including cinnamon soil, brown soil and dark brown soil.

\section{Research method}

As a city with rapid development, Xi'an has degraded the ecological environment of the construction land and affected the ecological suitability. Based on the characteristics of Xi'an city, this paper establishes an index system, and then analyses the influencing factors of urban construction land ecological adaptability according to the changes in the quantity and structure, and obtains the urban land use structure changes in Xi'an over the years. The benefit status, through remote sensing image analysis, obtained the ecological suitability evaluation map of Xi'an construction land.

\subsection{Factor selection and weight determination}

The weight of the evaluation factor is obtained by the Analytic Hierarchy Process (AHP). The principle is to establish a hierarchical structure model based on the logical structure relationship between the factors, and then compare the factors to each other, and analyze and judge by the expert scoring method. The relative importance of each evaluation index is established to obtain an evaluation matrix. The classification criteria and weights of construction land suitability evaluation factors are shown in table 1.

Table 1 Classification criteria and weights of construction land suitability evaluation factors

\begin{tabular}{cccc}
\hline Factor layer & Classification condition & Status description & Weights \\
\hline Elevation & $0 \sim 80$ & Very suitable & 0.1325 \\
& $80 \sim 120$ & More suitable & \\
Slope & $>20 \sim 200$ & Basically suitable & \\
& $<8^{\circ}$ & Not suitable & \multirow{2}{*}{0.1837} \\
$8^{\circ} \sim 16^{\circ}$ & Very suitable & \\
$16^{\circ} 25^{\circ}$ & More suitable & \\
Geomorphological & $>25^{\circ}$ & Basically suitable & \\
condition & Plain & Not suitable & \multirow{2}{*}{0.2305} \\
& Very suitable & \\
Land use status & Mountains & Not suitable & \\
& Residential land & Very unsuitable & \multirow{2}{*}{0.2411} \\
& dry land & Very suitable & \\
& Grassland & More suitable & \\
& Boodland & Not suitable & \\
Traffic status & Waters & Very unsuitable & \\
& Road and 500 m buffer & Very suitable & 0.0824 \\
& $500 \sim 1$ o00 m buffer & Basically suitable & \\
Built-up area & $>1000 \mathrm{~m}$ buffer & Not suitable & \\
& Urban built-up area & Very suitable & 0.1298 \\
& 2 km buffer & More suitable & \\
& $>2$ km buffer & Not suitable & \\
\hline
\end{tabular}




\subsection{Establishment of evaluation index system}

The multi-factor weighted evaluation results of Xi'an were obtained by superimposing various factors on the MCE platform combined with spatial analysis related modules. The spatial superposition analysis of GIS is carried out by the weight of each factor, which is obtained as shown in figure 1 , and the single factor evaluation chart of ecological suitability evaluation of construction land in Xi'an is obtained.

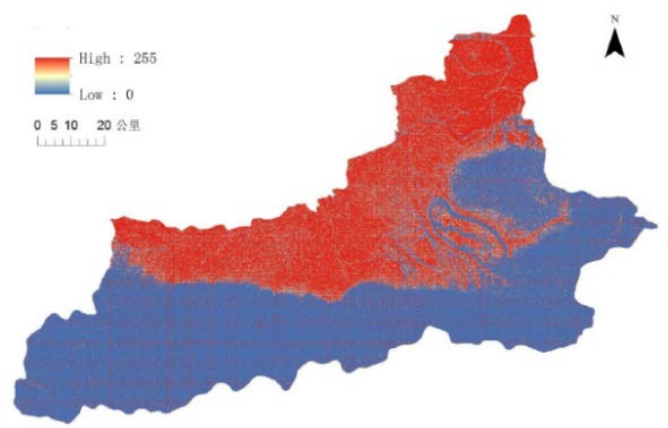

Slope influence factor

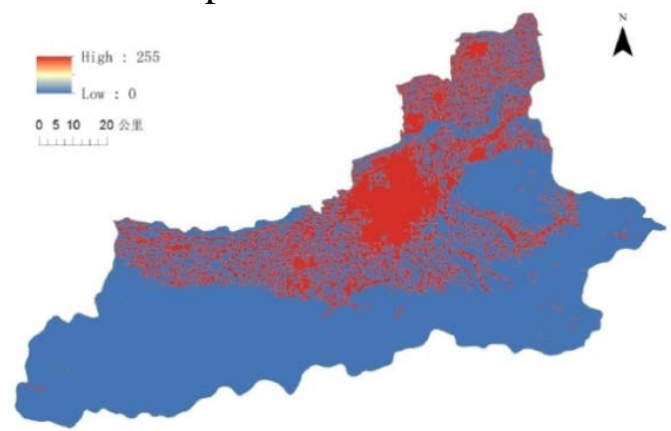

Distance factor from road

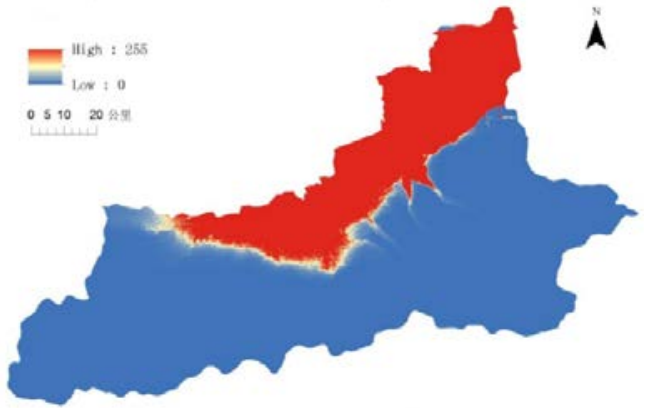

Elevation influence factor

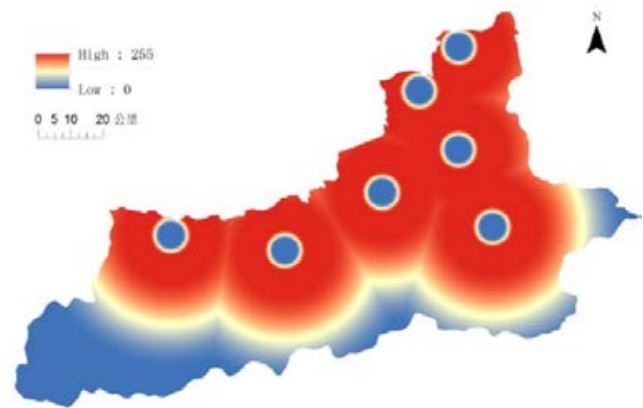

Distance factor from town center

Fig.1 Single factor evaluation

Using the selective weight linear combination method, the integrated limit factor after superposition of the spatial analysis module and the standardized impact factors are used to calculate the intersection, so as to generate the final Xi'an construction land conversion suitability evaluation map. The suitability of construction land, different grades and proportions are shown in table 2. The suitability map for construction land conversion are shown in figure 2 .

Table 2 Suitability of construction land, different grades and proportions

\begin{tabular}{ccc}
\hline Suitability & Area $/ \mathbf{k m}^{2}$ & Proportion/\% \\
\hline Very suitable & $194.03 \mathrm{~km}^{2}$ & $44.34 \%$ \\
More suitable & $148.18 \mathrm{~km}^{2}$ & $33.86 \%$ \\
Basically suitable & $84.33 \mathrm{~km}^{2}$ & $19.27 \%$ \\
Not suitable & $0.90 \mathrm{~km}^{2}$ & $2.49 \%$ \\
\hline
\end{tabular}




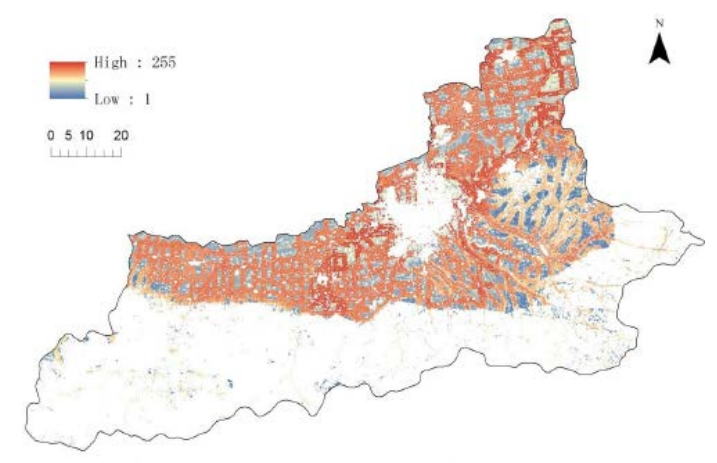

Figure 2 Suitability map for construction land conversion

\section{Conclusion}

The first type of area is suitable for land use, mainly distributed in the western part of Xi'an City. The terrain of this area is relatively flat, and the bearing capacity of the foundation is relatively high. It is suitable for building multi-storey buildings and above. The second type of area is a region with better suitability, which is mainly distributed in the southwestern part of Xi'an. The terrain of some areas is relatively large and undulating, which is suitable for the construction of low-rise and multi-storey buildings. The third type is the medium-suitability area, which mainly distributes within the city wall, around the city wall and within the 100-400 m range of Chang'an-Lintong fault. This area is not suitable for the land type with more intensive planning and construction personnel. The fourth type of land is the area with poor suitability, which mainly distributes near the cypress forest in the central urban area and within the distance of $100 \mathrm{~m}$ from the Chang'an-Lintong fault. This area is mainly located in the seismic fault zone, and its security is low, so it is not suitable for construction land.

In response to the evaluation results, the following suggestions are proposed: Relevant policies should be adopted to limit the excessive growth of construction land, make rational planning, maintain the amount of cultivated land, and limit the excessive development and occupation of woodland and wetland. Strengthening the environmental protection and preventing deterioration of ecological protection areas has become the most important work. At the same time, relevant policies should be formulated to improve the ecological awareness of regional residents.

\section{Acknowledgement}

Shaanxi social science fund project: Study on comprehensive measurement and spatial optimization of urban ecological bearing capacity of xi 'an city from the perspective of ecological civilization I (2014D18)

\section{References}

[1] Yu Yan, He Jianhua. Land eco-economic suitability evaluation based on niche suitability [J]. Journal of Agricultural Engineering, 2008, 24(1): 124-128.

[2] Qiu Bingwen, Chi Tianhe, Wang Qinmin, et al. Application and prospect of GIS in land suitability evaluation [J]. Geography and Geo-Information Science, 2004, 20(5): 20-23.

[3] Yang Shaojun, Liu Xiaofu, Shu Yumin. Theory and method of urban land ecological suitability evaluation [J]. Journal of Eco-Environment, 2009, 18(1): 380-385.

[4] Wang Xinjie, Xue Dongqian, Yan Junping, et al. Analysis of the relationship between urbanization and ecological environment in Xi'an[J]. Earth and Environment, 2010 (1): 43-48. 